\title{
Assessing the Efficiency of Robot Communication
}

\author{
K. A. Polshchykov ${ }^{a, *}$, S. A. Lazarev ${ }^{a, * *}$, I. S. Konstantinov ${ }^{a, * * *}$, O. N. Polshchykova ${ }^{a, * * * *}$, \\ L. F. Svoikina ${ }^{a, * * * * *}$, E. V. Igityan ${ }^{a, * * * * * *}$, and M. S. Balakshin ${ }^{a, * * * * * * *}$ \\ ${ }^{a}$ Belgorod State University, Belgorod, 308015 Russia \\ *e-mail:polshchikov@bsu.edu.ru \\ **e-mail:lazarev_s@bsu.edu.ru \\ ***e-mail: konstantinov@bsu.edu.ru \\ ****e-mail:polshchikova@bsu.edu.ru \\ *****e-mail: svojkina@bsu.edu.ru \\ ******e-mail: medvedeva_e@bsu.edu.ru \\ *******e-mail: 1337150@bsu.edu.ru
}

Received January 24, 2020; revised January 24, 2020; accepted January 24, 2020

\begin{abstract}
A model is proposed for estimating the efficiency of communication by robots capable of social interaction with humans. A generalized index of the robot's effectiveness in communication is derived. That index is calculated by means of a fuzzy deduction module.
\end{abstract}

Keywords: robot systems, communication functions, effectiveness, performance index, fuzzy-deduction module, Matlab, Simulink

DOI: $10.3103 / \mathrm{S} 1068798 X 20110155$

Today, robot systems are capable of a broad variety of tasks. In recent years, interest in social capabilities has been growing. Manufacturers of software and hardware have proposed robots capable of social interaction with humans [1, 2]. Examples include robot assistants that may perform domestic functions [3, 4]. The effectiveness of speech communication is very important here. How well the robot performs will depend ultimately on how comfortable with robot communication the human feels and how appropriately the robot behaves in whatever situations might arise.

In order to select social robots with the best communication capabilities and to identify necessary areas for improvement, we need means of assessing the effectiveness of robot communication with humans [5]. There is little literature on the development of such assessment procedures. Accordingly, models must be developed for assessing the effectiveness of communication by robots capable of social interaction with humans.

The effectiveness of robot communication is assessed on the basis of multiple criteria, taking account of the correctness and timeliness of robot speech with humans. It is expedient to use the generalized index $F$ of the effectiveness of robot communication.

The correctness of robot communication is related to its ability to conduct a reasonable dialog with a human. The timeliness of communication depends on the delay time of the robot in the communication process. Since these characteristics are not specific numerical values, their assessment requires the use of fuzzy sets: in the first case, high and low correctness of communication; in the second, high and low speed of response in communication [6].

In Matlab + Simulink software, the $\operatorname{Sys} F$ model is developed for determining the effectiveness of communication by the robot system (Fig. 1). The most important element of this model is the fuzzy-deduction module (Fuzzy_modul).

The elements $c 1-c 8$ are intended to input assessments of the correctness of robot responses to test queries. The values of these elements are input in accordance with the following logic: if the robot response to query $n$ is correct, then $c_{n}=1$; otherwise, $c_{n}=0$.

The elements $r 1-r 8$ determine the wait time $r_{n}$ in getting a response to specified query $n$ from the robot. For example, if $r_{4}=2.35$-that is, the robot responds after $2.35 \mathrm{~s}$ to test query 4 -then the value of element $r 4$ is 2.35 .

The element Calc_ $C$ of the model calculates the value of $C$, which is the aggregate correctness of the robot's responses to the test queries: $C=\sum_{n=1}^{N} c_{n}$. The elements Add, Calc $R$, and $N$ calculate $R$, which is the mean delay time of the virtual robot in responding to the test queries: $R=\frac{1}{N} \sum_{n=1}^{N} r_{n}$. Here $N$ is the number of test queries. 
The Fuzzy_modul element functions on the basis of the rules [7-9]

$$
\begin{aligned}
& \text { If }\left(C=H_{c}\right) \text { and }\left(R=H_{r}\right) \text {, then }\left(F=F_{1}\right) ; \\
& \text { if }\left(C=H_{c}\right) \text { and }\left(R=L_{r}\right) \text {, then }\left(F=F_{2}\right) ; \\
& \text { if }\left(C=L_{c}\right) \text { and }\left(R=L_{r}\right) \text {, then }\left(F=F_{4}\right) .
\end{aligned}
$$

Here the descriptors of robot communication are as follows: $H_{c}$ is the fuzzy set $<$ high correctness $>; L_{c}$ is the fuzzy set $\langle$ low correctness $\rangle ; H_{r}$ is the fuzzy set $\langle$ high speed of response $\rangle ; L_{r}$ is the fuzzy set $\langle$ low speed of response $\rangle$. The individual inferences from the fuzzy rules are as follows: $F_{1}=4, F_{2}=3$ and $F_{3}=2, F_{4}=1$.

The functions $C^{+}$and $C^{-}$characterizing the membership of $C$ in the fuzzy sets $H_{c}$ and $L_{c}$ and the functions $R^{+}$and $R^{-}$characterizing the membership of $r_{\text {in }}$ the fuzzy sets $H_{r}$ and $L_{r}$ are determined as follows

$$
\begin{aligned}
& C^{+}=\left\{\begin{array}{l}
0, \quad C<c_{0}^{+} ; \\
a_{1} C+b_{1}, \quad c_{0}^{+} \leq C<c_{1}^{+} ; \\
1, \quad C \geq c_{1}^{+} ;
\end{array}\right. \\
& C^{-}= \begin{cases}1, & C<c_{1}^{-} ; \\
a_{2} C+b_{2}, \quad c_{1}^{-} \leq C<c_{0}^{-} ; \\
0, & C \geq c_{0}^{-} ;\end{cases} \\
& R^{+}= \begin{cases}1, & R<r_{1}^{+} ; \\
a_{3} R+b_{3}, \quad r_{1}^{+} \leq R<r_{0}^{+} ; \\
0, & R \geq r_{0}^{+} ;\end{cases} \\
& R^{-}= \begin{cases}0, & R<r_{0}^{-} ; \\
a_{4} R+b_{4}, \quad r_{0}^{-} \leq R<r_{1}^{-} ; \\
1, & R \geq r_{1}^{-} ;\end{cases}
\end{aligned}
$$

here $c_{0}^{+}$and $c_{1}^{+}$are the boundaries of the internal segment of the graph of $C^{+} ; c_{0}^{-}$and $c_{1}^{-}$are the boundaries of the internal segment of the graph of $C^{-} ; r_{0}^{+}$and $r_{1}^{+}$ are the boundaries of the internal segment of the graph of $R^{+} ; r_{0}^{-}$and $r_{1}^{-}$are the boundaries of the internal segment of the graph of $R^{-} ; a_{1}$ and $b_{1}$ are the coefficients of the direct membership function $C^{+}$in the section $c_{0}^{+} \leq C<c_{1}^{+} ; a_{2}$ and $b_{2}$ are the coefficients of the direct membership function $C^{-}$in the section $c_{1}^{-} \leq C<c_{0}^{-}$; $a_{3}$ and $b_{3}$ are the coefficients of the direct membership function $R^{+}$in the section $r_{1}^{+} \leq R<r_{0}^{+}$; and $a_{4}$ and $b_{4}$ are the coefficients of the direct membership function $R^{-}$in the section $r_{0}^{-} \leq R<r_{1}^{-}$.

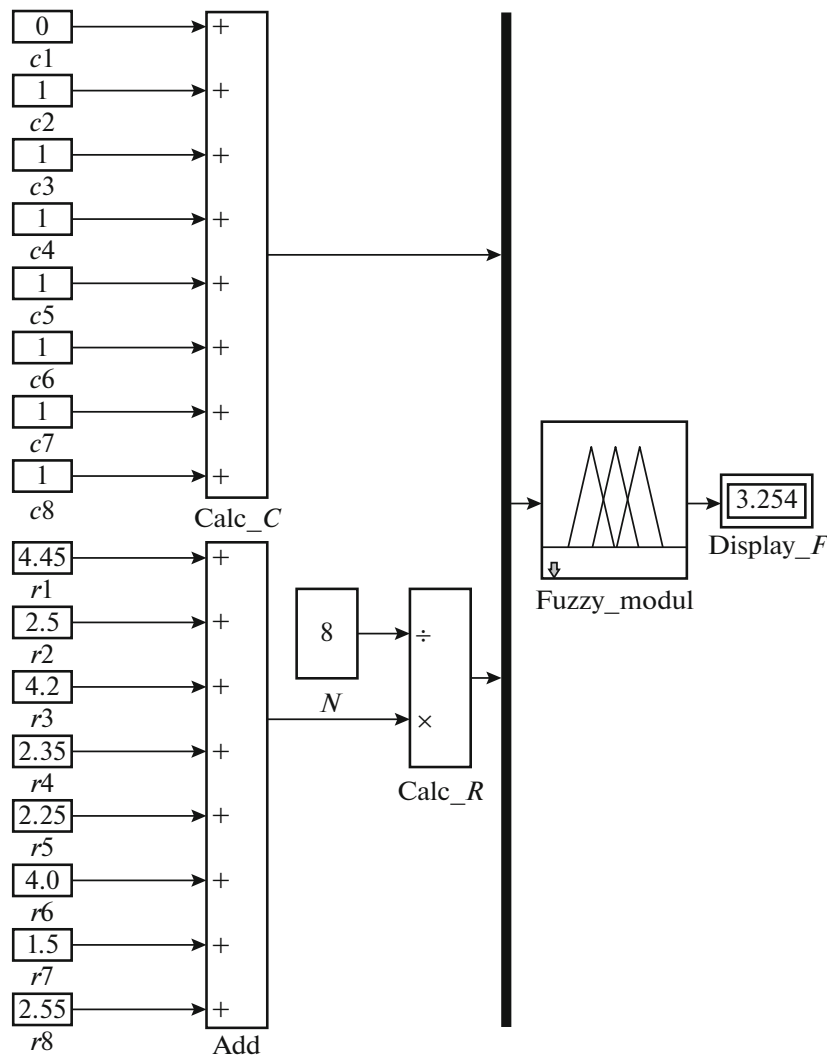

Fig. 1. Sys $F$ model for determining the effectiveness of communication by a robot system.

The generalized effectiveness of robot communication is calculated from the formula

$$
F=\frac{G_{1} Y_{1}+G_{2} Y_{2}+G_{3} Y_{3}+G_{4} Y_{4}}{G_{1}+G_{2}+G_{3}+G_{4}},
$$

where $G_{1}, G_{2}, G_{3}$, and $G_{4}$ are the results of aggregation calculated from the expressions $G_{1}=C^{+} \wedge R^{+}$; $G_{2}=C^{+} \wedge R^{-} ; G_{3}=C^{-} \wedge R^{+}$; and $G_{4}=C^{-} \wedge R^{-}$.

The Display_F module shows the results of calculating $F$.

Operation of the fuzzy-deduction system requires adjustment of the Fuzzy_modul element in accordance with $c_{0}^{+}, c_{1}^{+}, c_{0}^{-}, c_{1}^{-}, r_{0}^{+}, r_{1}^{+}, r_{0}^{-}$, and $r_{1}^{-}$. To that end, $K$ experiments are conducted with a virtual robot specially developed for adjustment of the fuzzy-deduction system. In each experiment $J$, experts must give a subjective estimate of the correctness of communication with the virtual robot and its speed of response.

\section{RESULTS OF COMPUTER EXPERIMENTS}

The proposed model is used in a series of computer experiments to assess the effectiveness of robot communication. As an example, we show the results of one 
Table 1

\begin{tabular}{c|c||c|c}
\hline Element & Value & Element & Value, $\mathrm{s}$ \\
\hline$c_{1}$ & 0 & $r_{1}$ & 4.45 \\
$c_{2}$ & 1 & $r_{2}$ & 2.5 \\
$c_{3}$ & 0 & $r_{3}$ & 4.2 \\
$c_{4}$ & 1 & $r_{4}$ & 2.35 \\
$c_{5}$ & 1 & $r_{5}$ & 2.25 \\
$c_{6}$ & 0 & $r_{6}$ & 4.0 \\
$c_{7}$ & 1 & $r_{7}$ & 1.5 \\
$c_{8}$ & 1 & $r_{8}$ & 2.55 \\
\hline
\end{tabular}

experiment in which the initial data for the elements $c 1-c 8$ and $r 1-r 8$ of the Sys $F$ model are as in Table 1.

On the basis of the experiments, the effectiveness of robot communication $F=3.245$ (shown by the Display_F element).

\section{CONCLUSIONS}

(1) In Matlab + Simulink software, a model has been developed for assessment of the effectiveness of communication by robots capable of social interaction with humans. The model is based on a fuzzy-deduction system taking account of the correctness and response time of the robot in communication.

(2) Computer experiments with this model yield the generalized effectiveness of robot communication $F$, which varies from 1 to 4 . Higher $F$ corresponds to more effective communication.

(3) The next step is to develop software on the basis of the proposed model and to test it experimentally.

\section{REFERENCES}

1. Social robots: high technology against loneliness. https://newtonew.com/science/sociable-robots-andhuman-loneliness. Accessed December 27, 2019.

2. Gaschler, A., Huth, K., and Giuliani, M., Modeling state of interaction from head poses for social humanrobot interaction. https://mediatum.ub.tum.de/doc/ 1285788/file.pdf. Accessed December 27, 2019.

3. Abdul Malik, N., Yussof, H., and Hanapiah, F.A., Potential use of social assistive robot based rehabilitation for children with cerebral palsy, Proc. 2nd IEEE Int. Symp. on Robotics and Manufacturing Automation, Roma, 2016, pp. 782-784.

4. Salem, M., Kopp, S., Wachsmuth, I., et al., Generation and evaluation of communicative robot gesture, Int. $J$. Soc. Rob., 2012, vol. 4, pp. 201-217.

5. Suga, Y., Ikuma, Y., Nagao, D., et al., Interactive evolution of human-robot communication in real world, Proc. 2005 IEEE/RSJ Int. Conf. on Intelligent Robots and Systems, Washington, DC: IEEE Comput. Soc., 2005, pp. 1438-1443.

6. Polshchykov, K.A., Lazarev, S.A., Polshchykova, O.N., and Igityan, E.V., The algorithm for decision-making supporting on the selection of processing means for big arrays of natural language data, Lobachevskii J. Math., 2019, vol. 40, no. 11, pp. 1831-1836.

7. Takagi, T. and Sugeno, M., Fuzzy identification of systems and its applications to modeling and control, IEEE Trans. Syst., Man Cybern., 1985, vol. 15, no. 1, pp. 116-132.

8. Konstantinov, I.S., Polshchykov, K.A., Lazarev, S.A., and Polshchykova, O.N., Model of neuro-fuzzy prediction of confirmation timeout in a mobile ad-hoc network, Proc. Int. Conf. "Mathematical and Information Technologies,” MIT-2016, Vrnjacka Banja, 2017, pp. 174-186.

9. Konstantinov, I.S., Lazarev, S.A., Polshchykov, K.A., and Mihalev, O.V., Theoretical aspects of evaluation of the corporative portal network traffic management, Int. J. Appl. Eng. Res., 2015, vol. 10, no. 24, pp. 4569145696.

Translated by B. Gilbert 